\title{
On the Intermediate Subgroup of the Gamma-Ray Bursts in the Swift Database
}

\author{
David Huja and Attila Mészáros \\ Astronomical Institute of the Charles University, V Holešovičkách 2, Prague, Czech Republic
}

\begin{abstract}
.
A sample of 286 gamma-ray bursts, detected by Swift satellite, is studied statistically by the $\chi^{2}$ test and the Student t-test, respectively. The short and long subgroups are well detected in the Swift data. But no intermediate subgroup is seen. The non-detection of this subgroup in the Swift database can be explained, once it is assumed that in the BATSE database the short and the intermediate subgroups form a common subclass.
\end{abstract}

Keywords: gamma-ray astrophysics, gamma-ray bursts

PACS: $01.30 . \mathrm{Cc}, 95.55 . \mathrm{Ka}, 95.85 . \mathrm{Pw}, 98.70 . \mathrm{Rz}$

\section{DATA SAMPLES}

According to our knowledge, gamma-ray bursts (GRBs) are the most powerful mysterious explosions the Universe has ever seen since the Big Bang. With Swift satellite, since November 20, 2004, we have a tool, which can solve the gamma-ray burst mystery. We define two samples from the Swift dataset [1]: The sample of GRBs without measured redshift $z$ (189 GRBs), and the sample with measured $z$ (97 GRBs). The Swift catalogue consists of the name of GRB, its BAT duration $T_{90}$, BAT fluence at range $15-150 \mathrm{keV}$, BAT peak flux at range $15-150 \mathrm{keV}$, and redshift. The sample covers the period November 2004 - December 2007; the first / last event is GRB041227 / GRB071227. We have studied both samples separately and also together as the whole sample (286 GRBs).

\section{$\chi^{2}$ FITTING OF THE DURATION FOR THE WHOLE SAMPLE}

The first evidence about the existence of three subgroups of GRBs came from the $\chi^{2}$ fitting of the durations of BATSE dataset [2]. We proceed here identically. We study the whole sample and the two ones. On the X-axis there are the bins (intervals) of decimal $\log T_{90}$, and on the $y$-axis there is the number of GRBs in each interval. The number of bins is 10 and the bins define a histogram. We fitted the histogram. The whole sample of the GRBs has 276 GRBs with the measured duration. The best fit of the whole sample with one single Gaussian curve gives: $\mu=1.42$ (with mean $T_{90}=26.30 \mathrm{~s}$ ), $\sigma=0.94, \chi^{2}=$ 53.54. The goodness of the fit with 8 dof (degrees of freedom) gives the rejection on the $99.99 \%$ significance level [3, 4]. The fit with the sum of two Gaussian curves gives: $\mu_{1}=-0.27\left(T_{90}(1)=0.54 \mathrm{~s}\right), \sigma_{1}=0.94, \mu_{2}=1.57$ $\left(T_{90}(2)=37.15 \mathrm{~s}\right), \sigma_{2}=0.56, w=0.12(12 \%$ of the GRBs belongs to the short GRBs), $\chi^{2}=6.72$. Here $\chi^{2} \simeq d o f$ $=5$ and we obtain an excellent fit with the significance level $50 \%$. The fit with the sum of three Gaussian curves gives: $\mu_{1}=0.30\left(T_{90}(1)=2.00 \mathrm{~s}\right), \sigma_{1}=1.27, w_{1}=0.20$, $\mu_{2}=0.82\left(T_{90}(2)=6.61 \mathrm{~s}\right), \sigma_{2}=0.07, w_{2}=0.12, \mu_{3}=$ $1.72\left(T_{90}(3)=52.48\right), \sigma_{3}=0.46, \chi^{2}=3.82$, we obtain an excellent fit for $d o f=2$, because the significance level is only $78 \%$. The decreasing of $\Delta \chi^{2}=2.89$ is not statistically significant [3, 4], and hence the introduction of the third intermediate subgroup is not necessary. The fit is shown on the Figure 3.

\section{$\chi^{2}$ FITTING OF THE DURATION FOR THE SAMPLE WITH MEASURED REDSHIFT}

The sample with measured redshifts contains 94 GRBs with measured duration. The best fit of the sample with one single Gaussian curve gives: $\mu=1.43\left(T_{90}=26.92 \mathrm{~s}\right)$, $\sigma=0.87, \chi^{2}=12.59$. The goodness of the fit with 8 dof does not reject the hypothesis of one single Gaussian curve. The rejection is only on the $85 \%$ significance level. The fit with the sum of two Gaussian curves gives: $\mu_{1}=-0.46\left(T_{90}(1)=0.35 \mathrm{~s}\right), \sigma_{1}=0.51, \mu_{2}=1.52\left(T_{90}(2)=\right.$ $33.11 \mathrm{~s}), \sigma_{2}=0.62, w=0.06$ (6\% of the GRBs belongs to the short GRBs), $\chi^{2}=3.45$. Here we obtain an excellent fit with the significance level $97 \%$ [3, 4], thus the fit can not be rejected. The fit with the sum of three Gaussian curves gives: $\mu_{1}=-0.71\left(T_{90}(1)=0.19 \mathrm{~s}\right), \sigma_{1}=0.70, w_{1}$ $=0.09, \mu_{2}=0.51\left(\chi^{2}(2)=3.24 \mathrm{~s}\right), \sigma_{2}=0.01, w_{2}=0.01$, $\mu_{3}=1.56\left(T_{90}(3)=36.31 \mathrm{~s}\right), \sigma_{3}=0.54, \chi^{2}=3.16$, it is an excellent fit with $d o f=2$, because the significance level is only $80 \%$. The decreasing of $\Delta \chi^{2}=0.29$ is not 


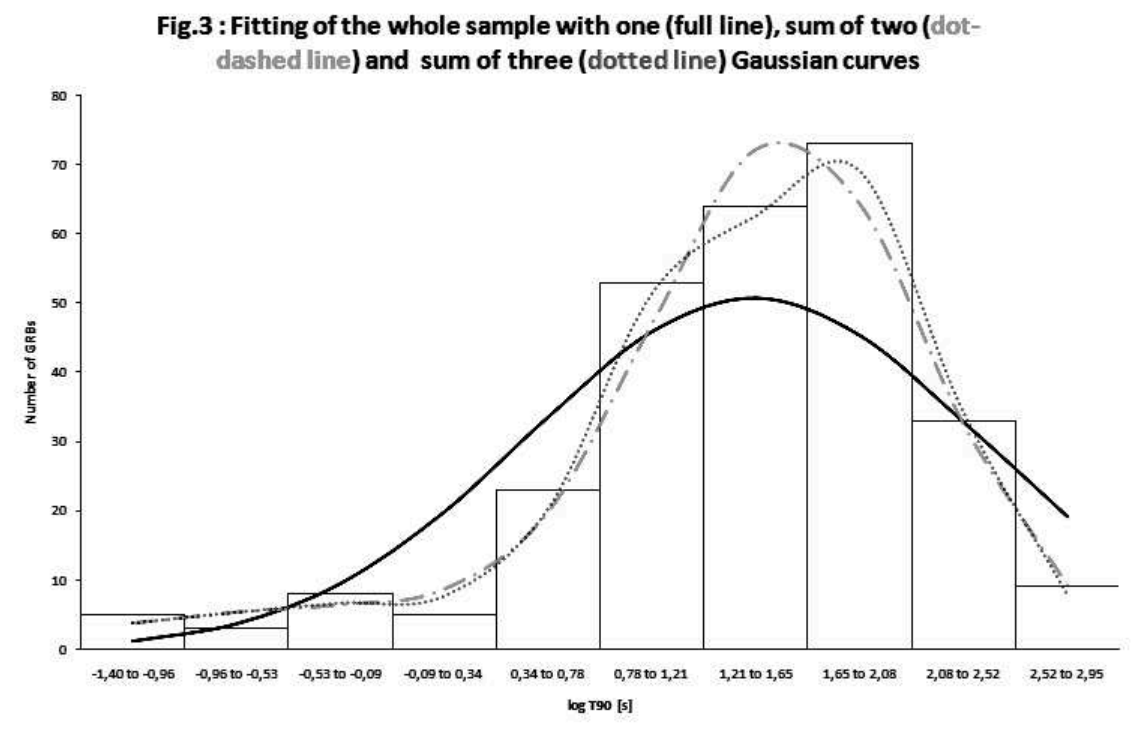

statistically significant [3, 4], also the introduction of the third intermediate subgroup is not necessary. The fit is shown on the Figure 2.

\section{$\chi^{2}$ FITTING OF THE DURATION FOR THE SAMPLE WITHOUT MEASURED REDSHIFT}

The sample without measured redshifts contains 182 GRBs with measured duration. The best fit of the sample with one single Gaussian curve gives: $\mu=1.40\left(T_{90}=\right.$ 25.12s), $\sigma=0.95, \chi^{2}=43.23$. The goodness of the fit with 8 dof gives the rejection on the $99.99 \%$ significance level. The fit with the sum of two Gaussian curves gives: $\mu_{1}=-0.73\left(T_{90}(1)=0.19 \mathrm{~s}\right), \sigma_{1}=0.71, \mu_{2}=1.54\left(T_{90}(2)=\right.$ $34.67 \mathrm{~s}), \sigma_{2}=0.56, w=0.09$ (9\% of the GRBs belongs to the short GRBs), $\chi^{2}=3.42$. Here we obtain an excellent fit, which can not be rejected, [3, 4]. The fit with the sum of three Gaussian curves gives: $\mu_{1}=-0.70\left(T_{90}(1)\right.$ $=0.20 \mathrm{~s}), \sigma_{1}=0.70, w_{1}=0.09, \mu_{2}=0.51\left(T_{90}(2)=\right.$ $3.24 \mathrm{~s}), \sigma_{2}=0.01, w_{2}=0.01, \mu_{3}=1.56\left(T_{90}(3)=36.31 \mathrm{~s}\right)$, $\sigma_{3}=0.34, \chi^{2}=3.16$, hence is an excellent fit with $d o f=2$, because the significance level is only $80 \%$. The decreasing of $\Delta \chi^{2}=0.26$ is not statistically significant [3, 4], and then the introduction of the third intermediate subgroup is not necessary. The fit is shown on the Figure 1.

\section{CONCLUSION}

Because the article [2] described the existence of the third (intermediate) subgroup of GRBs in the BATSE database by the $\chi^{2}$ fitting of the duration, we worked out an identical procedure on the existing Swift database. Contrary to the BATSE GRBs $([2,5,6])$, the Swift GRBs do not require any introduction of the third intermediate subgroup in contrast to [7]; the hypothesis is that the short and intermediate subgroups should form one single subclass.

\section{ACKNOWLEDGMENTS}

This study was supported by the GAUK grant No.46307, by the OTKA grant No.T48870, by the Grant Agency of the Czech Republic, grants No. 205/08/H005, and by the Research program MSM0021620860 of the Ministry of Education of the Czech Republic. The authors appreciate valuable discussion and help of J. Řípa.

\section{REFERENCES}

1. http://swift.gsfc.nasa.gov/docs/swift/archive/grb_tab

2. I. Horváth, ApJ 508, 757 (1998).

3. R.J. Trumpler \& H.F. Weaver 1953, Statistical Astronomy, University of California Press, Berkeley.

4. M.G. Kendall \& A. Stuart 1973, The advanced theory of statistics, Charles Griffin \& Co.Ltd.

5. I. Horváth, $A \& A$ 392, 791 (2002).

6. I. Horváth, $A \& A$ 447, 23 (2006).

7. I. Horváth, et al. $A \& A$ (in press), (2008), arXiv:0808.1067v2. 
Fig. 2: Fitting of the sample with $\underline{z}$ with one (full line), sum of two (dotdashed line) and sum of three (dotted line) Gaussian curves

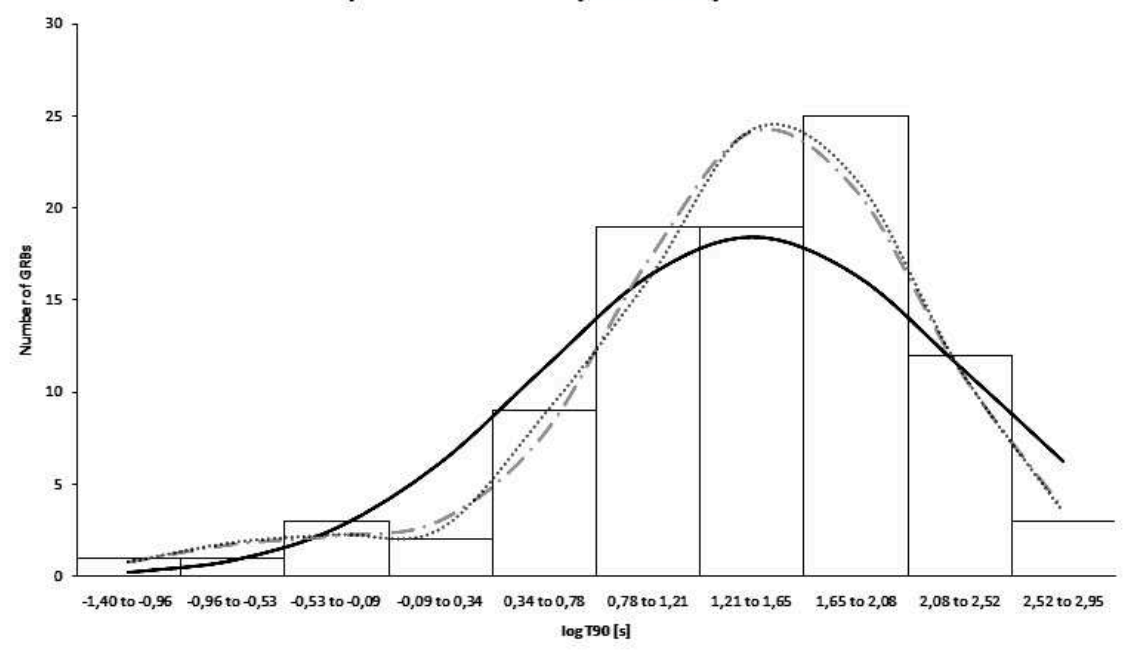

Fig. 1: Fitting of the sample without $\underline{z}$ with one (full line), sum of two (dotdashed line) and sum of three (dotted line) Gaussian curves

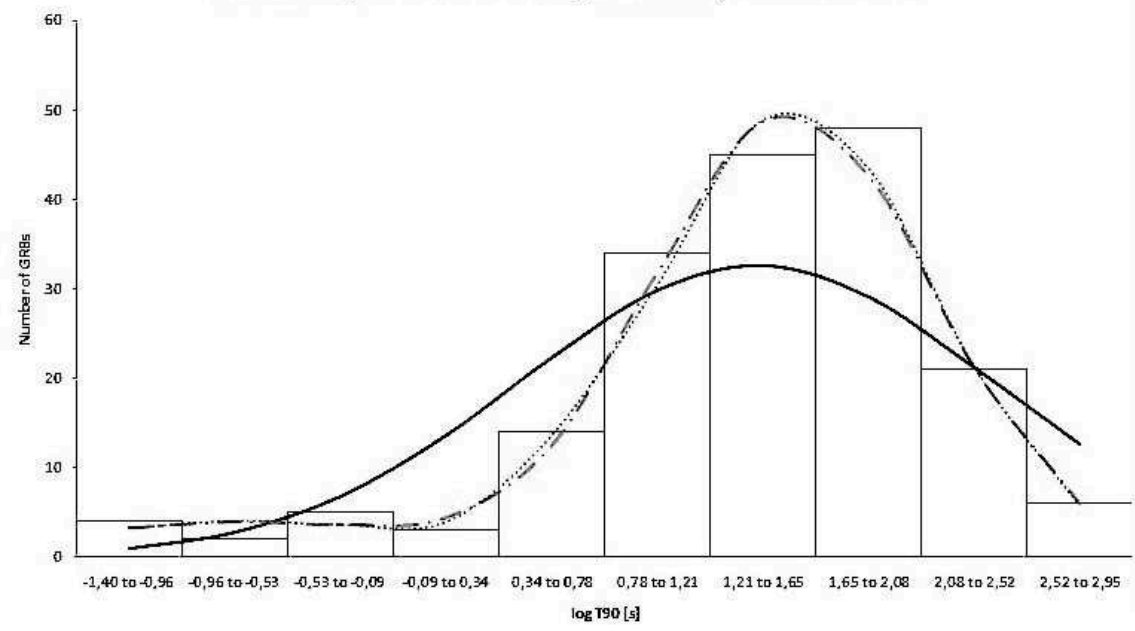

